\title{
Identifying Promising School-Based Intervention Programs to Promote 24-Hour Movement Guidelines among Children: Protocol for a Systematic Review
}

\author{
Javier Rodrigo-Sanjoaquín ${ }^{1,2, *(\mathbb{D})}$, Julien E. Bois ${ }^{2}$, Alberto Aibar Solana ${ }^{1}\left(\mathbb{D}\right.$, Léna Lhuisset ${ }^{2}(\mathbb{D}$ \\ and Javier Zaragoza Casterad ${ }^{1}$ (D) \\ 1 Department of Didactics of the Musical, Plastic and Corporal Expression, Faculty of Human Sciences and \\ Education, EFYPAF Research Group, University of Zaragoza, 22003 Huesca, Spain; aibar@unizar.es (A.A.S.); \\ zaragoza@unizar.es (J.Z.C.) \\ 2 Laboratory Movement, Equilibre, Performance, Santé (MEPS), Faculty of Sciences and Techniques of \\ Physical and Sports Activities (STAPS), Université de Pau \& Pays de l'Adour, 65000 Tarbes, France; \\ julien.bois@univ-pau.fr (J.E.B.); lena.lhuisset@univ-pau.fr (L.L.) \\ * Correspondence: jrodrigo@unizar.es or jrsanjoaquin@univ-pau.fr
}

Received: 9 October 2020; Accepted: 11 November 2020; Published: 13 November 2020

\begin{abstract}
Three key health behaviors, physical activity (PA), sedentary behavior (SB) and sleep (SLP), have been identified by the 24-h framework as movement behaviors. School-based interventions targeting these multiple health behaviors among children have the potential to increase health outcomes. Despite this, the efficacy and sustainability of school-based movement behavior interventions among children has not been evaluated yet. To fill this gap in literature, this systematic review will aim to: (1) Summarize and classify movement behavior strategies used in literature to improve PA, SB and SLP in/from school in children; and (2) measure the effect of movement behavior strategies used in literature to improve PA, SB and SLP in children. The review protocol was registered in PROSPERO (CRD42020199154). A systematic search will be conducted between 2010 to 2020 in five databases: Pubmed, Scopus, SPORTDiscuss, The Cochrane Library and Web of Science. Risk of bias and quality assessment will be evaluated and measured according to the recommended tools. This systematic review will provide information about which kind of school-based movement behavior interventions are effective, sustainable and the best to implement in children.
\end{abstract}

Keywords: 24-h guidelines; movement behaviors; physical activity; sedentary behavior; sleep; school; children; intervention program

\section{Introduction}

Public health concern has increased over the past decade due to the rise of non-communicable diseases as the first cause of death worldwide [1]. It is known that insufficient physical activity (PA) is one of the most important health behavioral risks for non-communicable diseases in children, youths, and especially in adults [1-3]. Sedentary behaviors (SB) such as sedentary screen-time behaviors (i.e., TV viewing, computer use, playing video-games, or smartphone use), and sitting time (i.e., leisure and occupational sedentary time) are behaviors closely related to physical inactivity in children, but must be considered separately [4,5]. Related to sleep (SLP) behavior, some studies have shown that unhealthy SLP (i.e., irregular sleep patterns, poor sleep quality and short sleep duration) is associated with a higher risk of obesity and a lack of health outcomes (e.g., good physical shape, physical and psychological well-being, healthy dietary patterns, and cognitive performance) among children and adolescents worldwide [6-8]. For convenience only the acronyms PA, SB and SLP instead of their full expressions will be used throughout the manuscript. 
Some research studies indicated that the adoption of a healthy lifestyle during childhood can have protective effects against the onset of chronic disease [9], and health-related behaviors acquired at this age usually tend to persist into adulthood [10], so it can influence sustainable long term health behaviors. PA, SB and SLP, separately and combined, can influence and increase health benefits in children [11,12]. These three health behaviors are correlated [13-16] and can interact among them to increase healthy outcomes (e.g., high PA/low SB/improve SLP) in children and youths [17]. Traditionally, school health research has been mainly focused on promoting PA and reducing SB in children [11], but SLP research has usually been treated independently and separately [18]. Although this approach has been very important to the field so far, emerging evidence indicates that today another integrated approach is necessary to understand and promote school health behaviors in children $[17,19]$. PA is an important health behavior, but only accounts for a small part of daily time, however SB and SLP make up the majority of a 24-hour period [20]. As a consequence, a new paradigm was developed in 2016, called the 24-hour Movement Guidelines [17,21]. This framework recognizes and integrates the importance of correctly combining PA, SB and SLP to reach and improve health outcomes in children [12]. According to the recommendations provided by the 24-h Movement Guidelines [17] and the WHO [22], children aged 5-12 years should accumulate $>60$ min of daily moderate-to-vigorous physical activity (MVPA), spend several hours in a day doing Light Physical Activity (LPA), sleep more than $9 \mathrm{~h}$ per night and dedicate less than $2 \mathrm{~h}$ each day to sedentary screen-time behaviors.

Previous studies suggested that combinations of behaviors (e.g., increase PA, reduce SB and improve SLP quality) can impact health in a different way that would not be explained by the effect of individual behaviors studied separately $[23,24]$. Interventions targeting a combination of multiple health behaviors are a promising method to improve several health outcomes [25]. This approach evidences that changing one health behavior could affect or improve others $[25,26]$. However, interventions that simultaneously address several health behaviors are usually difficult to implement at schools [26], especially due to various barriers and limitations (e.g., lack of teacher's formation in health promotion, a limited school timetable, the school curricula organization, etc.). On the other hand, interventions that combine PA (e.g., MVPA, LPA), SB (e.g., short sitting time, low screen-time) and SLP (e.g., high sleep quality, high sleep duration) have shown more beneficial outcomes compared with interventions that do not combine these behaviors $[12,20]$. School is an ideal setting for health promotion behaviors in children, because the majority of the population stay there for a long period of their lifetime, and children spend approximately $50 \%$ of their daily waking time at school [27]. Today, school children spend approximately $6-8 \mathrm{~h}$ per day at school, being sedentary $[28,29]$, so it is even more important to target school interventions that can increase PA (i.e., MVPA and LPA) and reduce SB (i.e., sitting time and screen time). Considering the 24-h movement guidelines framework, SLP should also be considered.

There are multiple reviews targeting PA [30-34], SB [27,35-38] and SLP [18,39-41] interventions separately, but there is a lack of reviews about multiple health behavior (i.e., 3 or more health behaviors) interventions. We have only found some reviews that target combined health behavior interventions (e.g., PA and SB, PA and SLP) in children that address issues such as obesity prevention [42-45], weight gain prevention [46], energy-balance [47], and preschoolers aged 0-4 years [48]. Other reviews related to multiple health behavior interventions (e.g., PA and SB, PA and SLP) have mainly focused on adolescents $[26,49-53]$ and suggested that although they were effective in terms of PA outcomes, more evidence and high quality studies are required to determine their effectiveness related to other health behaviors such as SB or SLP.

Some recent reviews have focused on examining effective PA and SB strategies $[45,54]$ and a recent umbrella review about school-based interventions to prevent weight gain in children has been published [46]. However, to the authors' knowledge, none of the previously published systematic reviews have exclusively dealt with the effectiveness of strategies related to movement behaviors (i.e., PA, SB and SLP) from multiple health behavior interventions in school children. As a consequence, to fill this gap in literature, this systematic review will aim to: (1) Summarize and classify movement behavior 
strategies used in literature to improve PA, SB and SLP in/from school in children; and (2) measure the effect of movement behavior strategies used in literature to improve PA, SB and SLP in children.

\section{Materials and Methods}

The protocol for this systematic review was conducted by the Preferred Reporting Items for Systematic Reviews and Meta-Analyses Protocols (PRISMA-P) guidelines [55], and it is available in Supplementary Material 1. This systematic review protocol was registered in PROSPERO (CRD42020199154), International Prospective Register of Systematic Reviews, and the report of this systematic review will be informed by PRISMA guidance [56]. Relevant modifications to this protocol will be indicated and published in the corresponding final systematic review.

\subsection{Eligibility Criteria}

To be eligible for the current systematic review, studies will be selected based on the following criteria. Studies must be published in a peer-reviewed English language journal, and target healthy school-children aged 5-12 years (i.e., primary school) without mental disabilities. Study participants are required to have a mean age of 5-11.99 years with at least two exposure measurement points, although follow-up measures of movement behaviors could happen past this age limit. The proposed systematic review will include interventional designs (i.e., randomized controlled trials, cluster randomized controlled trials and non-randomized studies [e.g., quasi-experimental studies, pilot studies and single group]) examining the influence of school-based interventions on movement behaviors. To be included in this systematic review, studies will be required to incorporate at least two movement behaviors from the three $24 \mathrm{~h}$ movement behaviors (i.e., PA and SB and SLP; PA and SB; PA and SLP; SB and SLP). These behaviors could be assessed by self-report (e.g., questionnaires) or device-based measures (e.g., accelerometer data, pedometers, etc.). According to the eligibility criteria of the recent SB review and meta-analysis conducted by Blackburn et al., [57], all outcomes relating to SB (i.e., leisure or occupational sitting time and sedentary screen-time behaviors) will be included. Furthermore, in line with another review protocol [58] and meta-analysis [26], studies addressing other health behaviors (e.g., dietary patterns, healthy nutrition, well-being, etc.), in addition to our movement behaviors of interest, will not be excluded for the review. Thus, in Figure 1, we show the logic model, as recommended in literature $[59,60]$ to map, inform, make more transparent, and ultimately describe the conceptualization of the protocol review. Finally, according to these recommendations [61], grey literature (e.g., book chapters, editorials, pre-prints, abstracts, congress communications, etc.) will not be included in the search strategy because grey literature is typically not published in peer-reviewed scholarly resources (i.e., journals), and could result in a large increase in the number of records to screen, with little added value for those that have already been identified.

\subsection{Search Strategy}

This systematic search will be conducted using the following electronic databases of peer-reviewed journal articles and online research registers: Pubmed, Scopus, SPORTDiscuss, The Cochrane Library and Web of Science, following the recommendations to conduct academic search systems for systematic reviews and meta-analyses [62]. The expected school-based strategies to be found in the intervention studies extracted across these databases search, will belong to specfic theoretical frameworks (i.e., Socio-ecological Model, Theory of Planned Behavior, Self-determination Theory, Social Cognitive Theory, Theory of Reasoned Action, Coordinated School Health Model, Intervention Mapping, Preceed-Proceed Model and Behavior Change Methodology) according to what is shown in Figure 1. Searches will be conducted for studies published between 2010 and October 2020, coinciding with the last decade. Eligible papers will be reviewed to identify other relevant potential studies that could be selected. Similarly, recent related systematic reviews will be consulted to identify any additional studies. The Population, Interventions, Comparisons, Outcomes and Study design (PICOS) framework [63] was followed to conduct the literature search (see Table 1). This strategy allows to 
identify key study concepts in the research question, like the logic model presented previously in Figure 1, and facilitate the search process [64]. Described search keywords are related to the following topics: target population (i.e., children), intervention (i.e., different strategies, techniques and health programs), and movement behaviors outcomes (i.e., PA, SB and SLP) in/from school. Search keywords will be combined with different Boolean operators (e.g., "AND", “OR", “NOT”). Selected keywords are based on previous systematic reviews that include some movement behaviors $[12,47,50,54,65]$. More detailed information about search strategy is available in Supplementary material 2.

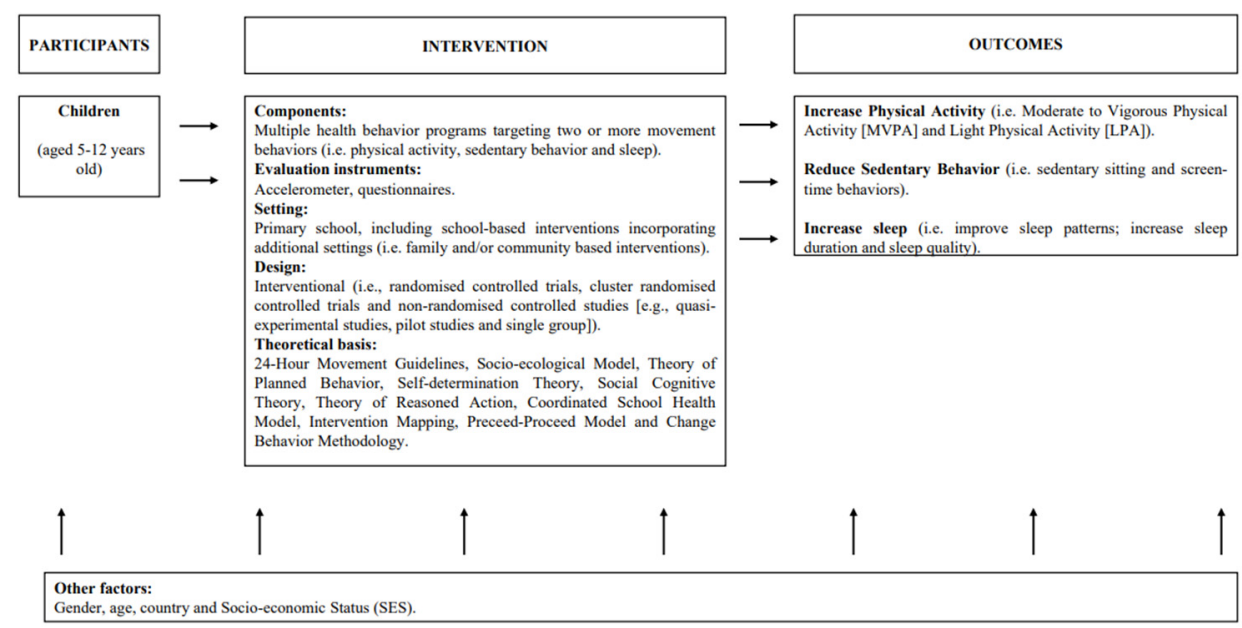

Figure 1. Logic model: School-based interventions targeting movement behaviors in children.

Table 1. PICO strategy: category, definition, and search terms.

\begin{tabular}{|c|c|c|}
\hline Category & Definition & Search Terms \\
\hline Population & $\begin{array}{l}\text { Children (from } 5 \text { to } \\
12 \text { years old). }\end{array}$ & $\begin{array}{l}\text { Child * OR student OR pupil OR infant OR childhood OR school * OR } \\
\text { schoolchildren OR school children OR schoolage * OR school-age * OR } \\
\text { school age * OR primary school OR elementary school OR basic school } \\
\text { NOT adolescent *. }\end{array}$ \\
\hline Intervention & $\begin{array}{l}\text { Health intervention } \\
\text { studies which use } \\
\text { different strategies } \\
\text { in/from school. }\end{array}$ & $\begin{array}{l}\text { strategy * OR technique * OR intervention * OR program * OR health } \\
\text { prevention OR health prevention program * OR health program * OR } \\
\text { health promotion program * OR health promotion intervention OR } \\
\text { health education OR health intervention OR school setting OR school } \\
\text { based intervention OR school-based intervention OR school based } \\
\text { program * OR school-based program * OR school program * OR school } \\
\text { health program OR school intervention OR school health intervention. }\end{array}$ \\
\hline Comparisons & Not applicable. & Not applicable. \\
\hline Outcomes & $\begin{array}{l}\text { Movement behaviors: } \\
\text { increase PA, reduce SB, } \\
\text { improve SLP. }\end{array}$ & $\begin{array}{l}\text { (PA OR Total PA OR MVPA OR VPA OR LPA OR total physical activity } \\
\text { OR moderate to vigorous physical activity OR vigorous physical } \\
\text { activity OR light physical activity OR physical activity OR physical } \\
\text { inactivity OR active OR activity * OR sport * OR sports participation OR } \\
\text { active transport OR active commuting OR leisure activity OR walking } \\
\text { OR aerobic exercise OR outdoor play OR exercise * OR motor behavior * } \\
\text { OR movement) AND (sedentart * OR sedentary behavior * OR } \\
\text { sedentary time OR sedentary lifestyle OR total sedentary time OR } \\
\text { sitting behavior * OR sitting time OR prolonged sitting OR domestic } \\
\text { activities OR computer use OR computer time OR media use OR video } \\
\text { games OR tablet use OR smartphone use OR mobile phone use OR } \\
\text { computer time OR television time OR TV time OR gaming OR screen } \\
\text { time OR screen-time OR reading OR TV viewing OR TV child room OR } \\
\text { television viewing OR video viewing) AND (sleep * OR sleep behavior * } \\
\text { OR sleep duration OR sleep quality OR sleep pattern * OR bedtime OR } \\
\text { sleep disturbance OR insomnia). }\end{array}$ \\
\hline
\end{tabular}

\footnotetext{
*: This sign allows to include all the endings or suffixes that these keywords may have when we search it.
} 


\subsection{Study Selection}

All identified studies from the literature search will be selected by two review authors in three steps as recommended in literature [61]. First, the titles and abstracts of the articles returned from the initial search will be screened and selected based on previous broken-down eligibility criteria. Second, full-text articles will be analyzed in detail and selected for eligibility. Third, the bibliographic references of all articles considered will be manually searched to identify relevant articles lost in the initial search strategy. If necessary, disagreements between the authors will be resolved by face-to-face discussion with the rest of authors to reach a consensus. Mendeley Citation Manager software will be used to store search results as well as to remove duplicate studies.

\subsection{Data Extraction}

Two review authors will independently and systematically extract data from included studies. The PRISMA guidance [56] will be used as a reference framework. Authors will identify the required information (i.e., publication details [i.e., authors, year], study characteristics [i.e., design, country, sample size, socio-economic status (SES), age and gender], movement behaviors targeted [i.e., PA, SB and SLP], theoretical framework [e.g., socio-ecological model, theory of planned behavior, etc.], intervention characteristics [i.e., delivery method, content and components], intervention frequency and duration [e.g., 3 intervention sessions/week during 14 weeks], stakeholders involved [i.e., teachers, families, community members, etc.], measurement tools [i.e., questionnaires, accelerometer data], main findings [related to outcomes measured] and follow-up if available). Possible future discrepancies between the authors will be solved by a consensus-based decision, or if necessary, a discussion with a third reviewer. If data clarifications are needed, authors of the original studies will be contacted. If necessary, authors from this protocol will make a maximum of two attempts of contact. All the extracted data will be synthesized using tables created with Microsoft Excel.

\subsection{Risk of Bias and Quality Assessment}

Some reviews $[61,66]$ indicated that literature generally failed to correctly identify and differentiate risk of bias from quality assessment. Thus, risk of bias refers to potential systematic errors that produce bias in final results. Quality assessment is the rigor and quality control in the research methodology and findings, which gives confidence in the results [67]. In that sense, two authors will independently assess the risk of bias of the selected studies using the "The Cochrane Risk of Bias Tool 2" (RoB-2) [68] for randomized studies, and "ROBINS-I" [69] for non-randomized studies. These tools cover a range of domains of potential bias, and any discrepancies between the raters will be resolved by a third reviewer. Scores will be summed across the domains evaluated to give a final total score of the risk of bias for each study. According to the aforementioned risk of bias tools [68,69], studies will be classified as "high risk" (i.e., if most items are rated with "some concerns", or at least one item is rated with "high risk"); as "some concerns" (i.e., if at least one item is rated with "some concerns"), and finally, as "low risk" (i.e., if all the items are rated as "low risk"). Related to quality assessment, the "Quality Assessment Tool for Before-After (Pre-Post) Studies with No Control Group" will be used to evaluate intervention studies without control group, and the "Quality Assessment of Controlled Intervention Studies" will be used [61] to evaluate intervention studies with control group. The "Quality Assessment Tool for Before-After (Pre-Post) Studies with No Control Group" integrates 12 items (NIH: https://www.nhlbi.nih.gov/health-topics/study-quality-assessment-tools) having the option to obtain a maximum score of 12 points. The "Quality Assessment Tool of Controlled Intervention Studies" integrates 14 items (NIH; https://www.nhlbi.nih.gov/health-topics/study-quality-assessment-tools) having the option to obtain a maximum score of 14 points. Each item will be rated as " 1 " when the information is reported or moderately reported, or " 0 " when the information is unclear or not reported. Intervention studies with no control group will be classified into "high quality" ( $>8$ points), "medium quality" (4-7 points), and "low quality" ( $<3$ points). Intervention studies with control group 
will be classified into "high quality" (>10 points), "medium quality" (5-9 points), and "low quality" ( $<4$ points). Two authors will independently assess each study, and discrepancies will be resolved by a third reviewer. We will not exclude studies based on findings from the quality assessment.

\subsection{Data Synthesis}

A narrative synthesis on all available data will be conducted. Depending on the results, authors will decide to conduct a meta-analysis or simply a qualitative synthesis. If only a qualitative synthesis is finally carried out, summary tables describing studies will be performed. Qualitative analysis will answer our first goal referenced in the background: "to summarize and classify movement behavior strategies used in the literature to improve PA, SB and SLP in/from school in children". These tables will include the general details of the studies, the intervention content (i.e., movement behaviors targeted and theoretical framework), the delivery method (i.e., school, family, teacher or children strategies and stakeholders involved), and the socio-ecological level stage (i.e., individual, interpersonal, organizational, community or policy). A second table will include methodological quality of studies (i.e., risk of bias and quality of assessment). If possible, a quantitative analysis (i.e., meta-analysis) of all defined movement behavior effects (i.e., pre-post, follow-up), where enough data is available, will also be conducted. Quantitative analysis will answer our second goal referenced in the background: "to measure the effect of movement behavior strategies used in literature to improve PA, SB and SLP in children". We anticipate a high degree of heterogeneity with respect to intervention types/lengths, reporting of outcomes and outcome measurements.

\section{Discussion}

The proposed systematic review will be the first to evaluate the school-based movement behavior interventions designed to promote healthy outcomes among children and their efficacy: Increase PA (i.e., MVPA, LPA), reduce SB (e.g., sitting and screen-time) and improve SLP (i.e., sleep patterns, duration and quality). This systematic review of recent and older studies will also allow us to obtain information about the degree of development and sustainability of interventions addressing movement behaviors among primary school students. Although there are some recent systematic reviews, which have examined health behavior interventions in children and adolescents, these were focused on preventing diseases or unhealthy behaviors (e.g., overweight, obesity, physical inactivity, alcohol and smoke consumption, etc.), instead of promoting health behaviors $[26,45,46,54]$. Consequently, there is a lack of reviews focused on targeting movement behavior interventions in children. Thus, this protocol provides a description of the future systematic review to be carried out, exploring this literature gap. According to our task to find multiple health behavior intervention studies, previous reviews [26,45] found that studies usually do not detail their strategies or methods of intervention, and further high quality research is needed. In order to do that, an important number of methodological unclear studies is expected to be found in our future search. In addition, a review and meta-analysis carried by Champion et al., [26] found that school-based multiple health behavior interventions were beneficial in increasing PA, and reducing screen time (i.e., SB), but concluded that effects were small and the overall quality of evidence was low (measured by GRADE [Grading of Recommendations Assessment, Development and Evaluation] framework). As mentioned in the introduction, combinations of health behaviors can impact health in a different way, which would be not explained by the effect of individual behaviors separately $[23,24]$. However, recent reviews have found that benefits of single health behavior interventions versus multiple health behavior interventions remained unclear $[45,70]$. Nevertheless, although there is no consensus in this regard, the present review will only include school interventions targeting at least two movement behaviors simultaneously to further study this topic.

Strengths of this review protocol come firstly from the detailed research guide (i.e., eligibility criteria and search strategy exposed) that will help future researchers to conduct other valid and reliable systematic reviews related to movement behavior interventions. Another methodological strength that should be highlighted in the current review is the detailed quality assessment and risk of bias procedure, 
according to recent recommendations to conduct systematic reviews [61]. Related to the limitations of this review, language restriction of published English written studies could limit the generalization of future results. Another limitation is that authors could be ignoring other databases [62] and do not identify more studies in the present systematic review.

\section{Conclusions}

The systematic review to be carried out will provide information about existing studies that have implemented school-based intervention programs targeting movement behaviors to increase health outcomes in children. It is expected that data extracted will be able to identify the most effective strategies and measure the intervention program effect according to our aim. Moreover, results of the present study will show a deeper understanding of which are the most effective and sustainable intervention programs to improve movement behaviors (i.e., PA, SB and SLP) in school-aged children. Finally, reviewing a broad range of adequate intervention programs to improve movement behaviors in/from school will provide information to researchers and practitioners of our field about which kind of interventions and strategies are the best to implement, related to each study design and program objectives.

Supplementary Materials: The following are available online at http://www.mdpi.com/2071-1050/12/22/ 9436/s1, Supplementary Material 1: PRISMA-P (Preferred Reporting Items for Systematic review and Meta-Analysis Protocols) 2015 checklist: recommended items to address in a systematic review protocol; Supplementary Material 2: Electronic search: databases and terms included.

Author Contributions: All authors conceptualized this study. J.R.-S., J.Z.C. and J.E.B. formulated the research objectives, whereas J.R.-S., A.A.S. and L.L. pointed out the inclusion and exclusion criteria under the supervision of the other authors. J.R.-S. developed the search strategy with the supervision of the other authors. J.R.-S. registered the systematic review in PROSPERO, piloted the first search strategy and drafted the protocol manuscript. All the authors reviewed and contributed to the final manuscript. All authors have read and agreed to the published version of the manuscript.

Funding: This study was funded by the University of Zaragoza and the Reference Research Group Educación Física y Promoción de la Actividad Física (EFYPAF). Additionally, this study was supported by the University of Pau and the Laboratory Mouvement, Equilibre, Performance et Santé (MEPS). In addition, J.R.-S. is supported by a Doctoral Research Fellowship from the Energy and Environment Solutions (E2S) project at the University of Pau (grant number: AAP2019-45).

Conflicts of Interest: The authors declare no conflict of interest.

\section{Abbrevations}

$\begin{array}{ll}\text { PA } & \text { Physical Activity } \\ \text { SB } & \text { Sedentary Behaior } \\ \text { SLP } & \text { Sleep } \\ \text { MVPA } & \text { Moderate-to-Vigorous Physical Activity } \\ \text { LPA } & \text { Light Physical Activity } \\ \text { PRISMA } & \text { Preferred Reporting Items for Systematic Reviews and Meta-Analysis } \\ \text { PRISMA-P } & \text { Preferred Reporting Items for Systematic Review and Meta-Analysis Protocols }\end{array}$

\section{References}

1. World Health Organization (WHO). Global Status Report on Non-Communicable Diseases. 2014. Available online: https://apps.who.int/iris/bitstream/handle/10665/148114/9789241564854_eng.pdf? sequence $=1$ (accessed on 30 July 2020).

2. Ding, D.; Lawson, K.D.; Kolbe-Alexander, T.L.; Finkelstein, E.A.; Katzmarzyk, P.T.; Van Mechelen, W.; Pratt, M. The economic burden of physical inactivity: A global analysis of major non-communicable diseases. Lancet 2016, 388, 1311-1324. [CrossRef]

3. Ezzati, M.; Riboli, E. Behavioral and dietary risk factors for non-communicable diseases. N. Engl. J. Med. 2013, 369, 954-964. [CrossRef] [PubMed] 
4. Ding, D.; Rogers, K.; van der Ploeg, H.; Stamatakis, E.; Bauman, A.E. Traditional and emerging lifestyle risk behaviors and all-cause mortality in middle-aged and older adults: Evidence from a large population-based Australian cohort. PLoS Med. 2015, 12, e1001917. [CrossRef] [PubMed]

5. Lynch, B.M.; Owen, N. Too much sitting and chronic disease risk: Steps to move the science forward. Ann. Intern. Med. 2015, 162, 146-147. [CrossRef] [PubMed]

6. Cappuccio, F.P.; Cooper, D.; D’Elia, L.; Strazzullo, P.; Miller, M.A. Sleep duration predicts cardiovascular outcomes: A systematic review and meta-analysis of prospective studies. Eur. Heart J. 2011, 32, 1484-1492. [CrossRef] [PubMed]

7. Chaput, J.P.; Dutil, C. Lack of sleep as a contributor to obesity in adolescents: Impacts on eating and activity behaviors. Int. J. Behav. Nutr. Phys. Act. 2016, 13, 103. [CrossRef]

8. Golley, R.K.; Maher, C.A.; Matricciani, L.; Olds, T.S. Sleep duration or bedtime? Exploring the association between sleep timing behaviour, diet and BMI in children and adolescents. Int. J. Obes. 2013, 37, 546-551. [CrossRef]

9. Gore, F.M.; Bloem, P.J.N.; Patton, G.C.; Ferguson, J.; Joseph, V.; Coffey, C.; Sawyer, S.M.; Mathers, C.D. Global burden of disease in young people aged 10-24 years: A systematic analysis. Lancet 2011, 377, $2093-2102$. [CrossRef]

10. Telama, R.; Yang, X.; Leskinen, E.; Kankaanpaa, A.; Hirvensalo, M.; Tammelin, T.; Viikari, J.S.; Raitakari, O.T. Tracking of physical activity from early childhood through youth into adulthood. Med. Sci. Sports Exerc. 2014, 46, 955-962. [CrossRef]

11. Chaput, J.P.; Carson, V.; Gray, C.E.; Tremblay, M.S. Importance of all movement behaviors in a $24 \mathrm{~h}$ period for overall health. Int. J. Environ. Res. Public Health 2014, 11, 12575-12581. [CrossRef]

12. Saunders, T.J.; Gray, C.E.; Poitras, V.J.; Chaput, J.-P.; Janssen, I.; Katzmarzyk, P.T.; Olds, T.; Gorber, S.C.; Kho, M.E.; Sampson, M.; et al. Combinations of physical activity, sedentary behaviour and sleep: Relationships with health indicators in school-aged children and youth. Appl. Physiol. Nutr. Metab. 2016, 41, S283-S293. [CrossRef] [PubMed]

13. Salmon, J.; Tremblay, M.S.; Marshall, S.J.; Hume, C. Health risks, correlates, and interventions to reduce sedentary behavior in young people. Am. J. Prev. Med. 2011, 41, 197-206. [CrossRef] [PubMed]

14. Tremblay, M.S.; Leblanc, A.G.; Kho, M.E.; Saunders, T.J.; Larouche, R.; Colley, R.C.; Goldfield, G.S.; Gorber, S.C. Systematic review of sedentary behaviour and health indicators in school-aged children and youth. Int. J. Behav. Nutr. Phys. Act. 2011, 8, 98. [CrossRef] [PubMed]

15. Uijtdewilligen, L.; Nauta, J.; Singh, A.S.; van Mechelen, W.; Twisk, J.W.; van der Horst, K.; Chinapaw, M.J. Determinants of physical activity and sedentary behaviour in young people: A review and quality synthesis of prospective studies. Br. J. Sports Med. 2011, 45, 896-905. [CrossRef] [PubMed]

16. Wu, X.Y.; Han, L.H.; Zhang, J.H.; Luo, S.; Hu, J.W.; Sun, K. The influence of physical activity, sedentary behavior on health-related quality of life among the general population of children and adolescents: A systematic review. PLoS ONE 2017, 12, e0187668. [CrossRef] [PubMed]

17. Tremblay, M.S.; Carson, V.; Chaput, J.P.; Connor Gorber, S.; Dinh, T.; Duggan, M.; Faulkner, G.; Gray, C.E.; Gruber, R.; Janson, K.; et al. Canadian 24-h movement guidelines for children and youth: An integration of physical activity, sedentary behaviour, and sleep. Appl. Physiol. Nutr. Metab. 2016, 41, S311-S327. [CrossRef] [PubMed]

18. Blunden, S.; Rigney, G. Lessons learned from sleep education in schools: A review of dos and don'ts. J. Clin. Sleep Med. 2015, 11, 671-680. [CrossRef]

19. Sallis, J.F. Needs and challenges related to multilevel interventions: Physical activity examples. Health Educ. Behav. 2018, 45, 661-667. [CrossRef]

20. Carson, V.; Tremblay, M.S.; Chaput, J.P.; Chastin, S.F. Associations between sleep duration, sedentary time, physical activity, and health indicators among Canadian children and youth using compositional analyses. Appl. Physiol. Nutr. Metab. 2016, 41, S294-S302. [CrossRef]

21. Department of Health. Australian 24-Hour Movement Guidelines for the Early Years. 2017. Available online: http://www.health.gov.au/internet/main/publishing.nsf/content/health-pubhlth-strateg-phys-actguidelines\#npa05 (accessed on 22 July 2020).

22. World Health Organization (WHO). Global Recommendations on Physical Activity for Health; World Health Organization: Geneva, Switherland, 2010. 
23. Chastin, S.F.; Palarea-Albaladejo, J.; Dontje, M.L.; Skelton, D.A. Combined effects of time spent in physical activity, sedentary behaviors and sleep on obesity and cardio-metabolic health markers: A novel compositional data analysis approach. PLoS ONE 2015, 10, e0139984. [CrossRef]

24. Chaput, J.P.; Saunders, T.J.; Carson, V. Interactions between sleep, movement and other non-movement behaviours in the pathogenesis of childhood obesity. Obes. Rev. 2017, 18, 7-14. [CrossRef] [PubMed]

25. Prochaska, J.J.; Spring, B.; Nigg, C.R. Multiple health behavior change research: An introduction and overview. Prev. Med. 2008, 46, 181-188. [CrossRef] [PubMed]

26. Champion, K.E.; Parmenter, B.; McGowan, C.; Spring, B.; Wafford, Q.E.; A Gardner, L.; Thornton, L.; McBride, N.; Barrett, E.L.; Teesson, M.; et al. Effectiveness of school-based eHealth interventions to prevent multiple lifestyle risk behaviours among adolescents: A systematic review and meta-analysis. Lancet Digital Health 2019, 1, e206-e221. [CrossRef]

27. Hegarty, L.M.; Mair, J.L.; Kirby, K.; Murtagh, E.; Murphy, M.H. School-based interventions to reduce sedentary behaviour in children: A systematic review. Aims Public Health 2016, 3, 520. [CrossRef] [PubMed]

28. Rush, E.; Coppinger, T.; Obolonkin, V.; Hinckson, E.; McGrath, L.; McLennan, S.; Graham, D. Use of pedometers to identify less active children and time spent in moderate to vigorous physical activity in the school setting. J. Sci. Med. Sport 2012, 15, 226-230. [CrossRef] [PubMed]

29. Van Stralen, M.M.; Yildirim, M.; Wulp, A.; Velde, S.J.T.; Verloigne, M.; Doessegger, A.; Androutsos, O.; Éva, K.; Brug, J.; Chinapaw, M.J.M. Measured sedentary time and physical activity during the school day of European 10-to 12-year-old children: The ENERGY project. J. Sci. Med. Sport 2014, 17, 201-206. [CrossRef]

30. Dobbins, M.; Husson, H.; DeCorby, K.; LaRocca, R.L. School-based physical activity programs for promoting physical activity and fitness in children and adolescents aged 6 to 18. Cochrane Database Syst. Rev. 2013, 2, 21-25. [CrossRef]

31. Jones, R.A.; Hinkley, T.; Okely, A.D.; Salmon, J. Tracking physical activity and sedentary behavior in childhood: A systematic review. Am. J. Prev. Med. 2013, 44, 651-658. [CrossRef]

32. Love, R.E.; Adams, J.; van Sluijs, E.M. Equity effects of children's physical activity interventions: A systematic scoping review. Int. J. Behav. Nutr. Phys. Act. 2017, 14, 134. [CrossRef]

33. Love, R.; Adams, J.; van Sluijs, E.M. Are school-based physical activity interventions effective and equitable? A meta-analysis of cluster randomized controlled trials with accelerometer-assessed activity. Obes. Rev. 2019, 20, 859-870. [CrossRef]

34. Metcalf, B.; Henley, W.; Wilkin, T. Effectiveness of intervention on physical activity of children: Systematic review and meta-analysis of controlled trials with objectively measured outcomes (EarlyBird 54). BMJ 2012, 345, e5888. [CrossRef] [PubMed]

35. Altenburg, T.M.; Kist-van Holthe, J.; Chinapaw, M.J. Effectiveness of intervention strategies exclusively targeting reductions in children's sedentary time: A systematic review of the literature. Int. J. Behav. Nutr. Phys. Act. 2016, 13, 65. [CrossRef] [PubMed]

36. Biddle, S.J.; Petrolini, I.; Pearson, N. Interventions designed to reduce sedentary behaviours in young people: A review of reviews. Br. J. Sports Med. 2014, 48, 182-186. [CrossRef] [PubMed]

37. Friedrich, R.R.; Polet, J.P.; Schuch, I.; Wagner, M.B. Effect of intervention programs in schools to reduce screen time: A meta-analysis. J. Pediatria 2014, 90, 232-241. [CrossRef] [PubMed]

38. Marsh, S.; Foley, L.S.; Wilks, D.C.; Maddison, R. Family-based interventions for reducing sedentary time in youth: A systematic review of randomized controlled trials. Obes. Rev. 2014, 15, 117-133. [CrossRef] [PubMed]

39. Blunden, S.L.; Chapman, J.; Rigney, G.A. Are sleep education programs successful? The case for improved and consistent research efforts. Sleep Med. Rev. 2012, 16, 355-370. [CrossRef]

40. Cassoff, J.; Knäuper, B.; Michaelsen, S.; Gruber, R. School-based sleep promotion programs: Effectiveness, feasibility and insights for future research. Sleep Med. Rev. 2013, 17, 207-214. [CrossRef]

41. Chung, K.F.; Chan, M.S.; Lam, Y.Y.; Lai, C.S.Y.; Yeung, W.F. School-Based Sleep Education Programs for Short Sleep Duration in Adolescents: A Systematic Review and Meta-Analysis. J. Sch. Health 2017, 87, 401-408. [CrossRef]

42. Agaronov, A.; Ash, T.; Sepulveda, M.; Taveras, E.M.; Davison, K.K. Inclusion of sleep promotion in family-based interventions to prevent childhood obesity. Child. Obes. 2018, 14, 485-500. [CrossRef] 
43. Ash, T.; Agaronov, A.; Aftosmes-Tobio, A.; Davison, K.K. Family-based childhood obesity prevention interventions: A systematic review and quantitative content analysis. Int. J. Behav. Nutr. Phys. Act. 2017, 14, 113. [CrossRef]

44. Khambalia, A.Z.; Dickinson, S.; Hardy, L.L.; Gill, T.A.; Baur, L.A. A synthesis of existing systematic reviews and meta-analyses of school-based behavioural interventions for controlling and preventing obesity. Obes. Rev. 2012, 13, 214-233. [CrossRef]

45. Lambrinou, C.-P.; on behalf of the Feel4Diabetes-study group; Androutsos, O.; Karaglani, E.; Cardon, G.; Huys, N.; Wikström, K.; Kivelä, J.; Ko, W.; Karuranga, E.; et al. Effective strategies for childhood obesity prevention via school based, family involved interventions: A critical review for the development of the Feel4Diabetes-study school based component. BMC Endocr. Disord. 2020, 20, 1-20. [CrossRef] [PubMed]

46. Goldthorpe, J.; Epton, T.; Keyworth, C.; Calam, R.; Armitage, C.J. Are primary/elementary school-based interventions effective in preventing/ameliorating excess weight gain? A systematic review of systematic reviews. Obes. Rev. 2020, 21, e13001. [CrossRef] [PubMed]

47. Anselma, M.; Chinapaw, M.J.; Kornet-van der Aa, D.A.; Altenburg, T.M. Effectiveness and promising behavior change techniques of interventions targeting energy balance related behaviors in children from lower socioeconomic environments: A systematic review. PLoS ONE 2020, 15, e0237969. [CrossRef] [PubMed]

48. Kuzik, N.; Poitras, V.J.; Tremblay, M.S.; Lee, E.Y.; Hunter, S.; Carson, V. Systematic review of the relationships between combinations of movement behaviours and health indicators in the early years (0-4 years). BMC Public Health 2017, 17, 849. [CrossRef] [PubMed]

49. Hale, D.R.; Fitzgerald-Yau, N.; Viner, R.M. A systematic review of effective interventions for reducing multiple health risk behaviors in adolescence. Am. J. Public Heal. 2014, 104, e19-e41. [CrossRef]

50. Hynynen, S.-T.; Van Stralen, M.M.; Sniehotta, F.F.; Araújo-Soares, V.; Hardeman, W.; Chinapaw, M.J.M.; Vasankari, T.; Hankonen, N. A systematic review of school-based interventions targeting physical activity and sedentary behaviour among older adolescents. Int. Rev. Sport Exerc. Psychol. 2016, 9, 22-44. [CrossRef]

51. MacArthur, G.J.; Caldwell, D.M.; Redmore, J.; Watkins, S.H.; Kipping, R.; White, J.; Chittleborough, C.; Langford, R.; Er, V.; Lingam, R.; et al. Individual-, family-, and school-level interventions targeting multiple risk behaviours in young people. Cochrane Database Syst. Rev. 2018, 10, CD009927. [CrossRef]

52. Jackson, C.A.; Henderson, M.; Frank, J.W.; Haw, S.J. An overview of prevention of multiple risk behaviour in adolescence and young adulthood. J. Public Health 2012, 34 (Suppl. 1), i31-i40. [CrossRef]

53. Prochaska, J.J.; Prochaska, J.O. A review of multiple health behavior change interventions for primary prevention. Am. J. Lifestyle Med. 2011, 5, 208-221. [CrossRef]

54. Jones, M.; Defever, E.; Letsinger, A.; Steele, J.; Mackintosh, K.A. A mixed-studies systematic review and meta-analysis of school-based interventions to promote physical activity and/or reduce sedentary time in children. J. Sport Health Sci. 2020, 9, 3-17. [CrossRef] [PubMed]

55. Moher, D.; Shamseer, L.; Clarke, M.; Ghersi, D.; Liberati, A.; Petticrew, M.; Shekelle, P.; Stewart, L.; PRISMA-P Group. Preferred reporting items for systematic review and meta-analysis protocols (PRISMA-P) 2015 statement. Syst. Rev. 2015, 4, 1-9. [CrossRef] [PubMed]

56. Moher, D.; Liberati, A.; Tetzlaff, J.; Altman, D.G. The PRISMA Group. Preferred reporting items for systematic reviews and meta-analyses: The PRISMA statement. PLoS Med. 2009, 6. [CrossRef]

57. Blackburn, N.; Wilson, J.J.; McMullan, I.I.; Caserotti, P.; Giné-Garriga, M.; Wirth, K.; Coll-Planas, L.; Alias, S.B.; Roqué, M.; Deidda, M.; et al. The effectiveness and complexity of interventions targeting sedentary behaviour across the lifespan: A systematic review and meta-analysis. Int. J. Behav. Nutr. Phys. Act. 2020, 17, 1-18. [CrossRef]

58. Champion, K.E.; Newton, N.C.; Spring, B.; Wafford, Q.E.; Parmenter, B.J.; Teesson, M. A systematic review of school-based eHealth interventions targeting alcohol use, smoking, physical inactivity, diet, sedentary behaviour and sleep among adolescents: A review protocol. Syst. Rev. 2017, 6, 1-7. [CrossRef] [PubMed]

59. Anderson, L.M.; Petticrew, M.; Rehfuess, E.; Armstrong, R.; Ueffing, E.; A Baker, P.R.; Francis, D.P.; Tugwell, P. Using logic models to capture complexity in systematic reviews. Res. Synth. Methods 2011, 2, 33-42. [CrossRef] [PubMed]

60. Rehfuess, E.A.; Booth, A.; Brereton, L.; Burns, J.; Gerhardus, A.; Mozygemba, K.; Oortwijn, W.; Pfadenhauer, L.M.; Tummers, M.; Van Der Wilt, G.-J.; et al. Towards a taxonomy of logic models in systematic reviews and health technology assessments: A priori, staged, and iterative approaches. Res. Synth. Methods 2018, 9, 13-24. [CrossRef] [PubMed] 
61. Gunnell, K.E.; Poitras, V.J.; Tod, D. Questions and answers about conducting systematic reviews in sport and exercise psychology. Int. Rev. Sport Exerc. Psychol. 2020, 13, 297-318. [CrossRef]

62. Gusenbauer, M.; Haddaway, N.R. Which academic search systems are suitable for systematic reviews or meta-analyses? Evaluating retrieval qualities of Google Scholar, PubMed, and 26 other resources. Res. Synth. Methods 2020, 11, 181-217. [CrossRef]

63. Schardt, C.; Adams, M.B.; Owens, T.; Keitz, S.; Fontelo, P. Utilization of the PICO framework to improve searching PubMed for clinical questions. BMC Med. Inform. Decis. Mak. 2007, 7, 16. [CrossRef]

64. Eriksen, M.B.; Frandsen, T.F. The impact of patient, intervention, comparison, outcome (PICO) as a search strategy tool on literature search quality: A systematic review. J. Med Libr. Assoc. 2018, 106, 420. [CrossRef] [PubMed]

65. Chong, K.H.; Parrish, A.M.; Cliff, D.P.; Kemp, B.J.; Zhang, Z.; Okely, A.D. Changes in physical activity, sedentary behaviour and sleep across the transition from primary to secondary school: A systematic review. J. Sci. Med. Sport 2019, 23, 498-505. [CrossRef] [PubMed]

66. Higgins, J.P.; Altman, D.G.; Gøtzsche, P.C.; Jüni, P.; Moher, D.; Oxman, A.D.; Savović, J.; Schulz, K.F.; Weeks, L.; Sterne, J.A.C.; et al. The Cochrane Collaboration's tool for assessing risk of bias in randomised trials. BMJ 2011, 343, d5928. [CrossRef] [PubMed]

67. Boutron, I.; Page, M.J.; Higgins, J.P.; Altman, D.G.; Lundh, A.; Hróbjartsson, A.; on behalf of the Cochrane Bias Methods Group. Considering bias and conflicts of interest among the included studies. Cochrane Handbook Syst. Rev. Interv. 2019, 177-204. [CrossRef]

68. Sterne, J.A.; Savović, J.; Page, M.J.; Elbers, R.G.; Blencowe, N.S.; Boutron, I.; Cates, C.J.; Cheng, H.-Y.; Corbett, M.S.; Eldridge, S.M.; et al. RoB 2: A revised tool for assessing risk of bias in randomised trials. BMJ 2019, 366, 14898. [CrossRef]

69. Sterne, J.A.; A Hernán, M.; Reeves, B.C.; Savović, J.; Berkman, N.D.; Viswanathan, M.; Henry, D.; Altman, D.G.; Ansari, M.T.; Boutron, I.; et al. ROBINS-I: A tool for assessing risk of bias in non-randomised studies of interventions. BMJ 2016, 355, i4919. [CrossRef]

70. James, E.L.; Freund, M.; Booth, A.; Duncan, M.J.; A Johnson, N.; E Short, C.; Wolfenden, L.; Stacey, F.G.; Kay-Lambkin, F.; Vandelanotte, C. Comparative efficacy of simultaneous versus sequential multiple health behavior change interventions among adults: A systematic review of randomised trials. Prev. Med. 2016, 89, 211-223. [CrossRef]

Publisher's Note: MDPI stays neutral with regard to jurisdictional claims in published maps and institutional affiliations.

(C) 2020 by the authors. Licensee MDPI, Basel, Switzerland. This article is an open access article distributed under the terms and conditions of the Creative Commons Attribution (CC BY) license (http://creativecommons.org/licenses/by/4.0/). 